\title{
A Novel Interface for Variable Flow Nanoscale LC/MS/MS for Improved Proteome Coverage
}

\author{
Johannes P. C. Vissers* \\ LC Packings, Baarsjesweg, Amsterdam, The Netherlands
}

\section{R. Kevin Blackburn and M. Arthur Moseley}

Proteomic Technologies, Genetics Research Division, GlaxoSmithKline, Research Triangle Park, North Carolina, USA

\begin{abstract}
A variable flow "peak trapping" liquid chromatography (LC) interface has been developed for the coupling of nanoscale LC to electrospray ionization mass spectrometry (ESI-MS). The presented peak trapping LC interface allows for the extended analysis time of co-eluting compounds and has been employed for the identification of proteins via tandem mass spectrometry (MS/MS). The variable flow process can be controlled either manually or in a completely automated manner where the mass spectrometer status determines the status of the variable flow interface. When the mass spectrometer operates in MS survey mode, the interface is operated in a so-called "high-flow" mode. Alternatively, the interface is operated in a "low-flow" mode during MS/MS analysis. In the "high-flow" mode of the variable flow process the column flow rate is typically around $200 \mathrm{~nL} / \mathrm{min}$, whereas in the "low-flow" mode the column effluent is introduced into the source of the mass spectrometer at $25 \mathrm{~nL} / \mathrm{min}$. In addition to the flow reduction during MS/MS analysis, the gradient is paused to preserve the peptide separation on the analytical nanoscale LC column. The performance of the variable flow nanoscale LC/MS/MS interface is demonstrated by the automated analysis of standard peptide mixtures and protein digests utilizing variable flow, data-dependent scanning MS/MS techniques, and automated database searching. (J Am Soc Mass Spectrom 2002, 13, 760-771) (C) 2002 American Society for Mass Spectrometry
\end{abstract}

$\mathrm{T}$ The field of protein mass spectrometry has undergone a major paradigm shift over the past several years, moving from the analysis of individual proteins to the analysis of total digests of complex protein mixtures (also know as shotgun proteomics). The development of shotgun proteomics by Yates [1-3] couples nanoscale LC for peptide analysis $[4,5]$, automated MS to MS/MS data acquisition software and hardware on modern tandem mass spectrometers [6], along with automated database searching software of modern search engines $[7,8]$. Shotgun proteomics has significantly increased the information content of protein mass spectrometry experiments, while at the same time significantly reducing the total analysis time required to analyze a complex protein mixture. Improvements in sample sensitivity and proteome coverage have been observed with this approach, compared to

Published online June 11, 2002

Address reprint requests to: Dr. M. Arthur Moseley, Proteomic Technologies, Genetics Research Division, GlaxoSmithKline, P.O. Box 13398, Research Triangle Park, NC 27709-3398. E-mail: mam30082@ glaxowellcome.com

*Present address: Micromass Europe, Transistorstraat 18, 1322 CE Weesp, The Netherlands. the "traditional" approach of using 2-D gels to separate the proteins, followed by in-gel digestion and analysis of the resulting peptides by mass spectrometry [3]. The shift in approach from the MS and MS/MS analysis of individual proteins to the analysis of complex mixtures of proteins has lead to the most significant figure of merit in proteomic analysis becoming the extent of proteome coverage obtained. Analytical methods that increase the coverage of the proteins in a sample will increase the impact of proteomics on biology.

For a typical $50 \mathrm{kD}$ protein, one would expect to have on the order of 40-50 tryptic peptides. Characterization of the complex mixture of peptides resulting from a total digest of a complex protein mixture requires the mass spectrometric analysis of hundreds to many thousands of peptides. Clearly, the MS and MS/MS analysis of a sample comprised of hundreds to thousands of proteins is challenging. Modern mass spectrometers with data dependent scanning software are capable of acquiring MS and MS/MS data from many hundreds of peptides per hour. Therefore, peptides must be delivered to the ion source at a rate compatible with the rate of acquisition of MS/MS data. Precursor ions are commonly selected for interrogation by MS/MS on the basis 
of their intensity, mass-to-charge ratio $(\mathrm{m} / \mathrm{z})$ and charge state. With ESI, the most common ion source used in these studies, the rate of delivery of peptides to the ion source must also take into account the fact that peptides will most often be presented in the form of multiple charge states. Thus, if each peptide is present in two or more charge states, the data acquisition problem is further exacerbated. This quite often leads to the same peptide being interrogated by MS/MS in most or all of the different charge states in which it is presented to the mass spectrometer.

There are three principal options for modulating the liquid chromatographic rate of delivery of peptides to the mass spectrometer-decreasing the slope of the chromatographic gradient, using comprehensive multidimensional chromatography (LC/LC) [3, 9-13], and modulating the flow of the chromatographic gradient (variable flow) [14-18]. The most straightforward method is to decrease the slope of the gradient used to elute peptides from the chromatographic column. With gradient slopes less than $0.15 \%$ per minute, one approaches isocratic elution conditions, with the resultant band broadening that is inherent in the isocratic elution of highly retained analytes (also known as the general elution problem). This limits the utility of this approach for modulating the rate of delivery of peptides to the ion source. Comprehensive multidimensional liquid chromatography is the most powerful method for modulating the rate of delivery of peptides to the mass spectrometer. The technique of LC/LC for peptide analysis is most commonly performed by using ion exchange chromatography to create tens of fractions of peptides, followed by reversed phase chromatographic analysis of each of these fractions. Thus, for LC/LC the rate of delivery of peptides to the mass spectrometer can be modulated by one to two orders of magnitude. This approach has found widespread use in shotgun proteomics, and has been applied to the analysis of thousands of proteins from a single mixture [13].

The third method for modulation of the rate of delivery of peptides to the mass spectrometer using variable flow was developed by Davis and Lee [14, 19]. Their system varied the flow rate of the mobile phase eluting from the reverse phase nanoscale LC column by varying the mobile phase pressure at the head of the column. Their work demonstrated the analysis of peptides from proteolytic digests of proteins at flow rates as low as tens of nanoliters/minute, with nanoscale LC/MS and MS/MS limits of detection as low as 10 femtomoles (injected onto the column). This variable flow approach facilitates the analysis of co-eluting peptides by decreasing the mobile phase flow rate of the LC column as the peptides are eluting in the chromatographic peak by a factor of five to ten. This increases the time available for the acquisition of MS/MS spectra from the peptides by a corresponding amount, leading to improved proteome coverage. It should be noted that this approach couples the principal advantages of nanoscale LC with the advantages of nanospray/ MS/MS [20, 21].

This paper describes an alternate approach to variable flow analysis, termed "peak trapping." In this system, the chromatographic eluent from a $75 \mu \mathrm{m}$ inner diameter (ID) nanoscale LC column flows at 200 nanoliters/minute through a microswitching valve to a fused silica transfer line connected to the spray tip of the ESI ion source. The volume of the transfer line and spray tip is approximately equal to the volume of a chromatographic peak from a $75 \mu \mathrm{m}$ ID nanoscale LC column (100 nanoliters). At the time that the mass spectrometer begins to detect peptides in the leading edge of a chromatographic peak, the remainder of the chromatographic peak is contained in the transfer line post-valve. Switching of the mass spectrometer from MS to MS/MS via automated data dependent scanning triggers the valve to switch, connecting the transfer line to a pressure vessel which delivers mobile phase at a flow rate of 25 nanoliters/minute. Thus, the majority of the chromatographic peak is delivered to the electrospray ion source at a low rate of 25 nanoliters per minute, an eightfold decrease in flow rate, providing up to an eightfold increase in MS/MS data acquisition time. Also, during this 'low-flow' mode the gradient progression is paused, preserving the chromatographic integrity of the separation of the analytes still retained on the column. When the mass spectrometer switches from MS/MS to MS acquisition, the valve returns to the original position, directly coupling the LC column to the transfer line, and the gradient restarts from the point at which the system entered 'low-flow' mode. This 'peak trapping' interface has been fully automated and coupled with a fully automated nanoscale LC/ MS/MS system, using a hybrid quadrupole time-offlight tandem mass spectrometer. It has been successfully applied for the shotgun proteomics analysis of a total digest of the 28S subunit of the bovine mitochondrial ribosome, which contains approximately 30 ribosomal proteins and $10+$ copurified contaminating proteins. In this real world analysis, the peak trapping variable flow interface gave a 2 - to 6 -time increase in the number of MS/MS spectra compared with the same system operated only in 'normal-flow' mode.

\section{Experimental}

\section{Chemicals and Reagents}

Peptide and protein standards, buffers, and other reagents were obtained from Sigma Chemical Company (St. Louis, MO). Endoproteinase Lys-C was from Wako Chemical Company (Richmond, VA), and trypsin from Roche Molecular Biochemicals.

\section{Sample Preparation}

Solution tryptic digests of thyroglobulin were carried out according to the manufacturer's protocols. In situ 
Lys-C digests of the $28 \mathrm{~S}$ subunit were performed according to the procedure of Burkhart [22]. The 28S subunit from mammalian mitochrondial ribosomes was purified from bovine liver as previously described [23].

\section{Nanoscale LC Instrumentation}

A FAMOS micro autosampler (LC Packings, Amsterdam, The Netherlands) with two additional built-in six-port valves was used for sample injection, sample clean-up, and preconcentration. Sample preconcentration and desalting was performed with a stand-alone LC pump that was operated isocratically at a flow-rate of $25 \mu \mathrm{L} / \mathrm{min}$. Cartridge type precolumns (LC Packings) with a length of $1 \mathrm{~mm}$ and an ID of $300 \mu \mathrm{m}$ were used to preconcentrate and desalt samples. The preconcentration column was filled with a $5 \mu \mathrm{m}, 100 \AA$ C18 PepMap $^{\mathrm{TM}}$ stationary phase (LC Packings). The loading solvent for sample preconcentration and clean-up consisted of an aqueous $0.1 \%$ formic acid solution.

All LC experiments were performed with an UltiMate $^{\mathrm{TM}}$ (LC Packings) nanoscale LC system [24]. The nanoscale LC outlet flow was transferred directly to one of the column switching valves of the micro autosampler to which the analytical column was connected using $25 \mu \mathrm{m}$ ID fused silica capillaries. Samples were separated on a $15 \mathrm{~cm} \times 75 \mu \mathrm{m}$ ID nanoscale LC column (LC Packings) packed with $3 \mu \mathrm{m}, 100 \AA$ C18 PepMap ${ }^{\mathrm{TM}}$ stationary phase at a flow rate of $200 \mathrm{~nL} / \mathrm{min}$. The gradient was developed from $5 \%$ to $40 \%$ acetonitrile for all the nanoscale LC/MS/MS experiments unless stated otherwise. Mobile Phase A consisted of $0.1 \%$ formic acid in acetonitrile/water (2:98, v/v) and mobile Phase $\mathrm{B}$ of $0.1 \%$ formic acid in acetonitrile/water $(80: 20, \mathrm{v} / \mathrm{v})$. UV absorption detection was performed at $214 \mathrm{~nm}$ with a $3 \mathrm{~nL}, 10 \mathrm{~mm}$ longitudinal Z-shaped flow cell (LC Packings).

\section{Variable Flow Nanoscale LC Interface}

The variable flow nanoscale LC interface consisted of an electrically activated 6-port Micro Injection Valve (Upchurch Scientific, Oak Harbor, WA, USA) that was positioned between the column and the ionization source of the mass spectrometer. The extremely small bore-to-bore volume of this valve (approximately $54 \mathrm{~nL}$ ) allows this valve to be used post-column without introducing significant extracolumn bandbroadening. A diagram of the valve set up is shown in Figure 1.

The column outlet was connected to a segment of 10

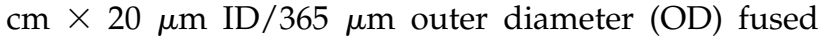
silica tubing using a MicroTight Union (Upchurch), which was subsequently connected to port one of the valve. Another segment of $20 \mu \mathrm{m}$ ID/365 $\mu \mathrm{m}$ OD fused silica was used to connect the valve to the spray needle. In the "high-flow" mode-that is, $200 \mathrm{~nL} / \mathrm{min}$ - the column effluent is directed through the valve and introduced into the source of the mass spectrometer.
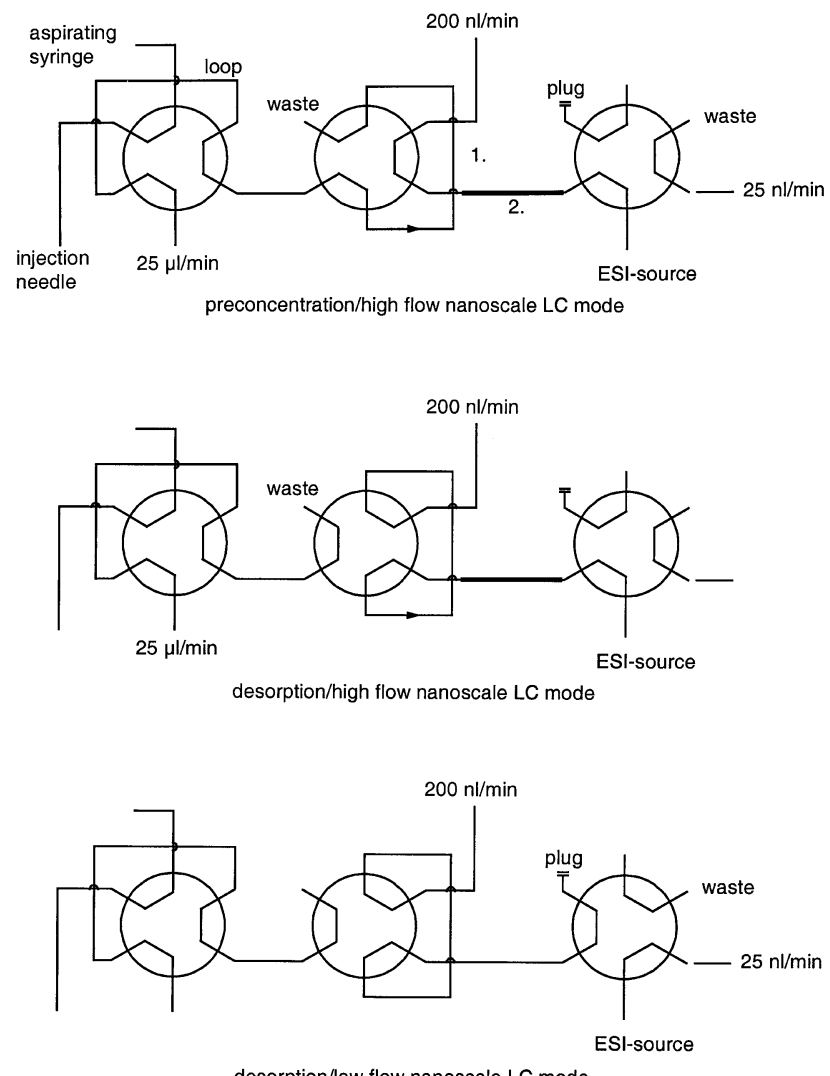

Figure 1. Flow diagram of system valves: (a) analyte preconcentration/desalting onto trapping cartridge. (b) analyte desorption and 'high flow' (200 nL/minute) analysis. (c) analyte desorption and 'low flow' (25 nL/minute) analysis. See "Experimental" section for a detailed discussion.

Port 6 of the valve was blocked with a piece of melted $360 \mu \mathrm{m}$ OD PEEK or fused silica tubing. Hence, when the valve is switched, the column effluent is blocked with excess pump flow diverting to waste through the precolumn flow splitter of the UltiMate. A 'pressure bomb' [4] was used to introduce the contents of the transfer line from Port 2 (transfer line volume set equal to the volume of a chromatographic peak at $200 \mathrm{~nL} /$ $\mathrm{min}$ ) to the mass spectrometer. The make-up solvent consisted of $0.1 \%$ formic acid in methanol/water (80:20, $\mathrm{v} / \mathrm{v}$ ) and was delivered to the valve at $25 \mathrm{~nL} / \mathrm{min}$. Hence, the introductory flow rate to the mass spectrometer was reduced by a factor of 8 in the "low-flow"

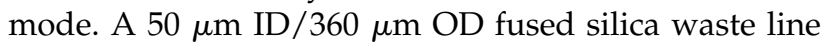
was connected to Port 4 of the valve.

In the "low-flow" mode, the gradient was paused to maintain integrity of the chromatographic separation, and the pump flow rate reduced to maintain a constant column pressure and to minimize spray instability in the switch from "low-flow" back to "high-flow." Both the variable flow nanoscale LC interface and the pump were controlled by means of contact closure inputs. A home-made interface generated a contact closure when the collision gas was turned on in the switch from MS to 
the MS/MS mode and vice versa, which toggled the valve and paused/continued the gradient. A more detailed description of the variable flow nanoscale LC interface is given in the section "Results and Discussion."

\section{Mass Spectrometry Instrumentation}

All MS experiments were performed with a Q-TOF ${ }^{\mathrm{TM}}$ hybrid quadrupole/time-of-flight mass spectrometer (Micromass Ltd., Manchester, UK) equipped with a Z-spray ion source, which has been described in detail previously [6]. The instrument consists of an electrospray ionization source, a quadrupole mass filter operating as a variable bandpass device, a hexapole collision cell, and an orthogonal acceleration time-of-flight (TOF) mass analyzer. The TOF mass analyzer is used to acquire data in both MS and MS/MS modes. A dual stage microchannel plate is used to detect ions and the output is sampled with a time-to-digital converter operated at $1 \mathrm{GHz}$. Spectra were collected at a resolution of approximately 5,000.

Nanoscale LC/MS/MS data was collected using data-dependent scanning, that is, automated MS to MS/MS switching. Two different modes of data-dependent scanning were evaluated: (i) the use of three collision energies for each precursor ion, with the selection of collision energies being used based on the $\mathrm{m} / \mathrm{z}$ of the precursor, or (ii) use of one collision energy for each precursor, with the collision energy used based on the charge state and the $m / z$ of the precursor ion.

The LC/MS electrospray interface was constructed in-house and consists of a stainless steel block acting as a spray tip mount, which was mounted on the xyz-stage of the mass spectrometer. A distally coated fused silica spray tip (New Objective, Cambridge,

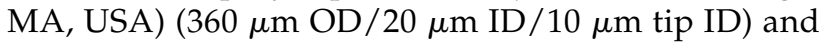
was held on the mount by means of a small clamp. The tip was positioned approximately $2 \mathrm{~mm}$ from the sampling cone of the electrospray source. Doublesided conductive tape was place in between the tip and the mount to insure electrical contact. The spray tip was typically held at $2-3 \mathrm{kV}$ and was connected to the UV flow cell outlet capillary or the variable flow nanoscale LC interface by means of low pressure dual diameter Teflon connectors (LC Packings) or MicroTight unions (Upchurch).

\section{Data Processing and Data Analysis}

All nanoscale LC/MS/MS data was processed automatically with the ProteinLynx (Micromass) module of the data acquisition software of the mass spectrometer. Mascot database searching software (Matrix Science Ltd., London, UK) was employed for peptide and protein identification [8].

\section{Results and Discussion}

\section{Variable Flow Nanoscale LC Interface}

The complete valve set up-including the variable flow nanoscale LC interface-is shown in Figure 1. The valve switching process consists of the following sequential steps. During the first step the loop content of the injection valve (Valve 1 ) of the autosampler is displaced onto the precolumn at a flow rate of $25 \mu \mathrm{L} / \mathrm{min}$. During this sample displacement step tryptic peptides are concentrated and hydrophilic and ionic contaminants removed. The desalting/preconcentration time used was $6 \mathrm{~min}$. Meanwhile the flow rate through the analytical $75 \mu \mathrm{m}$ ID nanoscale LC column is maintained at 200 $\mathrm{nL} / \mathrm{min}$.

After clean-up, the preconcentration valve (Valve 2) is switched and the precolumn placed in-line with the analytical column. Bound peptides are backflushed from the precolumn onto the analytical column by means of the gradient and separated on the analytical column. The flow rate through the analytical column during this stage of the separation process is 200 $\mathrm{nL} / \mathrm{min}$.

Switching of the variable flow nanoscale LC interface valve (Valve 3 ) is triggered by the mass spectrometer. A contact closure output is provided to the electrical actuator of the variable flow nanoscale LC interface valve when the mass spectrometer switches from the MS survey mode to the MS/MS data acquisition mode as peptides are detected eluting from the column. At the same time a contact closure is provided to the LC pump to pause the gradient, preserving the integrity of the chromatographic separation during low flow mode. Switching of the variable flow nanoscale LC interface valve also blocks the column flow, precluding the loss of analytes from the column during the "low-flow" mode. To prevent build-up of excessive system pressure and minimize spray instability in the switch back to "high-flow" mode, the pump flow is reduced to approximately $75 \%$ of its initial value. Excess pump flow is diverted pre-column to waste through the flow splitter mechanism of the UltiMate. Had the pump flow rate merely been dropped from 200 to $25 \mathrm{~nL} / \mathrm{min}$ to achieve "variable flow" conditions, analytes would have completely eluted into the mass spectrometer before the flow would have equilibrated to $25 \mathrm{~nL} / \mathrm{min}$ due to the time required for the pressure to relax across the length of the analytical column.

Upon switching to the variable flow interface valve, components detected by the mass spectrometer in the leading edge of the chromatographic peak are now contained in the transfer line between the variable flow nanoscale LC interface valve and the mass spectrometer. These components also are now being introduced into the source at a flow rate of $25 \mathrm{~nL} / \mathrm{min}$. When MS/MS data acquisition on a chromatographic peak is completed (based on MS/MS signal intensity falling below a certain threshold or acquisition time exceeding 


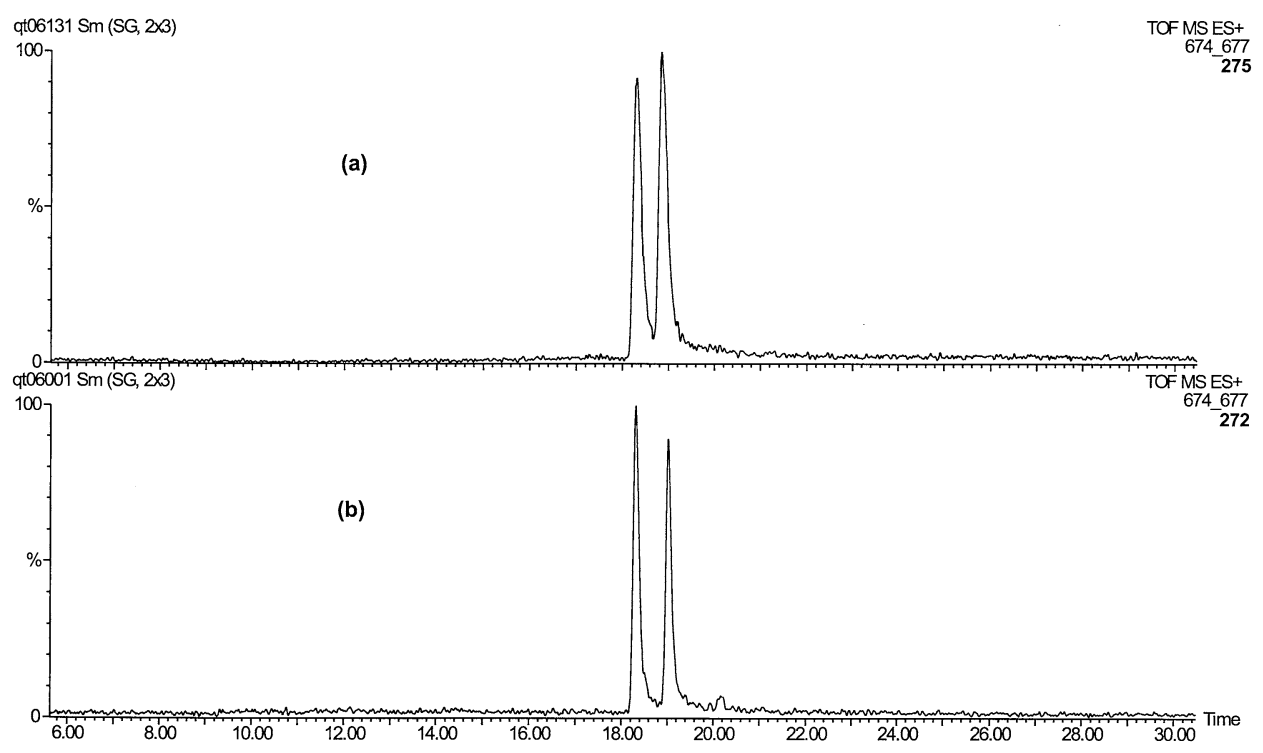

Figure 2. LC/MS separation of substance $P$ and substance $P$ (free acid) at $200 \mathrm{~nL} /$ minute with (a) and without (b) the variable flow post column valve. Placing the valve in-line (without switching) leads to a 3-second increase in the chromatographic peak width at half height (from 9 seconds to 12 seconds).

a user defined length of time), another contact closure output to the variable flow nanoscale LC interface valve and the pump of the LC system restores pump flow back to $200 \mathrm{~nL} / \mathrm{min}$, continues the gradient, and switches the variable flow nanoscale LC interface valve back such that the analytical column is again in-line with the mass spectrometer. The gradient progresses over the column at $200 \mathrm{~nL} / \mathrm{min}$ until the next component(s) is/are detected and the process is repeated. The complete valve switching process is automated and can be run unattended.

The extracolumn bandbroadening introduced by the post-column variable flow switching valve was found to be acceptable. This is illustrated by the results shown in Figure 2, where the separation of Substance P and Substance P (free acid) is shown using "normal flow" nanoscale LC conditions. Comparing both traces (mass chromatograms from $\mathrm{m} / \mathrm{z} 674$ to $\mathrm{m} / \mathrm{z} 677$ ), the average peak width at half height was observed to be approximately $9 \mathrm{~s}$ without the variable flow postcolumn valve interface (Figure $2 \mathrm{~b}$ ) and approximately $12 \mathrm{~s}$ with the variable flow postcolumn valve interface (Figure 2a) at a constant flow rate of $200 \mathrm{~nL} / \mathrm{min}$. For variable flow experiments an increase in peak width by a factor of 1.35 is not problematic since the peaks of interest are going to be spread out by a factor of eight in low-flow mode anyway. In fact, for normal, constant flow nanoscale LC/MS/MS, the interface can be left in-line since it does not significantly affect the quality of the separation obtained.

The type of variable flow nanoscale LC chromatography presented here has a number of advantages/ features compared to other variable flow techniques: (i) the column is not subjected to any dramatic pressure shocks; (ii) the integrity of the chromatographic sepa- ration is maintained; (iii) there are no losses of analytes from the column; (iv) the valve switching process is fully automated; (v) the variable flow nanoscale LC interface is suitable for any microcolumn LC systems as well as conventional, large column ID LC systems.

\section{Spray Tip Position Optimization}

In a recent paper, Geromanos et al. [25] showed that the position and the potential across the ion spray needle is of critical importance with respect to achieving the highest electrospray signal strength at very low infusion flow rates, i.e., $<25 \mathrm{~nL} / \mathrm{min}$. With the exception that a different MS source configuration and higher flow rates are being used in this study, such flow rate effects are expected. The goal in this study was to balance the response of the mass spectrometer between the two flow modes. An infusion experiment was conducted with $\left[\mathrm{Glu}^{1}\right]$-Fibrinopeptide $B$ using the variable flow interface that allowed switching alternatively between $200 \mathrm{~nL} / \mathrm{min}$ and 25 $\mathrm{nL} / \mathrm{min}$ where the LC was replaced with a syringe pump (data not shown). The position of the needle and the capillary voltage were carefully optimized to give a slightly higher response in the survey scan MS mode since the ability to detect an ion for automated data dependent scanning proved to be the most crucial aspect of the method. Results are presented elsewhere [26]. As an alternative to using a single capillary voltage, one could use optimized capillary for the two distinct flow rate regimes, which is facilitated by a recent release of the mass spectrometer operating software. 


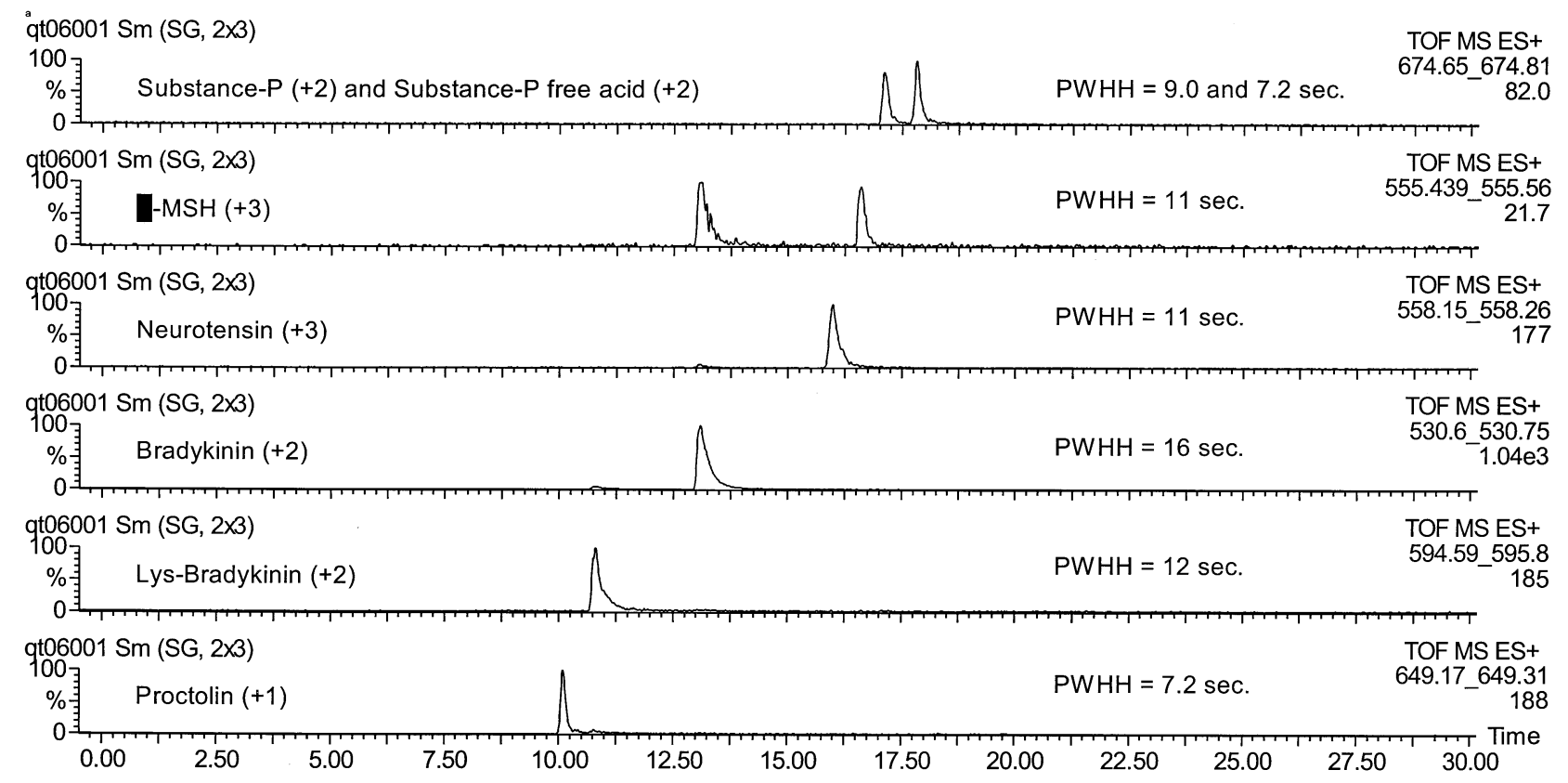

Figure 3. Selected ion chromatograms of a standard mixture of basic peptides acquired at 200 $\mathrm{nL} /$ minute. The peptides were selected as a stringent test of peak tailing in the variable-flow interface due to both chromatographic dead volumes and adsorption onto valve and tubing.

\section{Comparison between Constant Flow and Variable Flow Nanoscale LC/MS}

A peptide mixture consisting of seven basic peptides was used to evaluate the performance of the variable flow interface. The selected ion chromatograms of the individual components of the mixture were acquired with a constant flow of $200 \mathrm{~nL} / \mathrm{min}$ are shown in Figure 3. As can been seen, the basic nature of the peptides translates itself directly into the fact that the peaks are relatively broad and all exhibit some tailing. Note that the variable flow interface was installed in-line, which contributes to some extent to the tailing and broadening of the peaks as well.

The same mixture was analyzed with variable flow nanoscale LC/MS, of which the results are shown in Figure 4. The increase in peak width is due to lowering the introductory flow rate by a factor of eight during peak elution into the source of the mass spectrometer, allowing a factor of 8 times more time for co-eluting analytes to be interrogated by means of MS/MS. Note the bottom trace of Figure 4, where peaks eluting between 14 to 16 minutes were allowed to elute at constant flow of $200 \mathrm{~nL} / \mathrm{min}$, i.e., the variable flow was temporarily locked into the normal flow position. Although the variable flow nanoscale LC interface had been active to interrogate prior eluting analytes, the peak width did not increase significantly. Hence, the chromatographic resolution is maintained during variable flow mode since the column flow is stopped and the gradient paused.

To demonstrate the value of extended peak analysis time on a hybrid quadrupole/time-of-flight mass spectrometer, the results for neurotensin will be highlighted. Figure 5 shows the variable flow mass chromatogram (Figure 5a) and the constant flow mass chromatogram (Figure 5b). The increase in potential MS/MS acquisition time (based on the peak width at half height) was found to be equal to 6.4, whereas the instrumental configuration should have given a theoretical increase of a factor of 8 . This difference can be explained by the fact that the switching for this comparison was initiated manually. The panes on the right of Figure 5 show single scan spectra of the peak taken with variable flow (A1) and constant flow (B1). Since electrospray is a concentration sensitive technique [27], the total ion counts should be comparable for both normal and low flow modes. This was indeed observed to be the case, with observed ion counts of 189 and 150 for the "low-flow" mode and the "normal-flow" modes, respectively. The panes on the right of Figure 5 also show summed spectra across the width of the chromatographic peaks with both variable flow (A2) and constant flow (B2), with observed total ion counts of 2.52e 4 and 3.33e3, respectively, an increase of 6.6 (over the constant flow experiment) in the variable flow experiment. Here the true potential of variable flow nanoscale LC is demonstrated showing both increased MS/MS interrogation time with sensitivity comparable to that obtained in normal-flow mode. Hence, the amount of information in terms of the number of MS/MS spectra that can be assessed from one chromatographic peak can be significantly enhanced without degradation in the sensitivity of the measurement. 


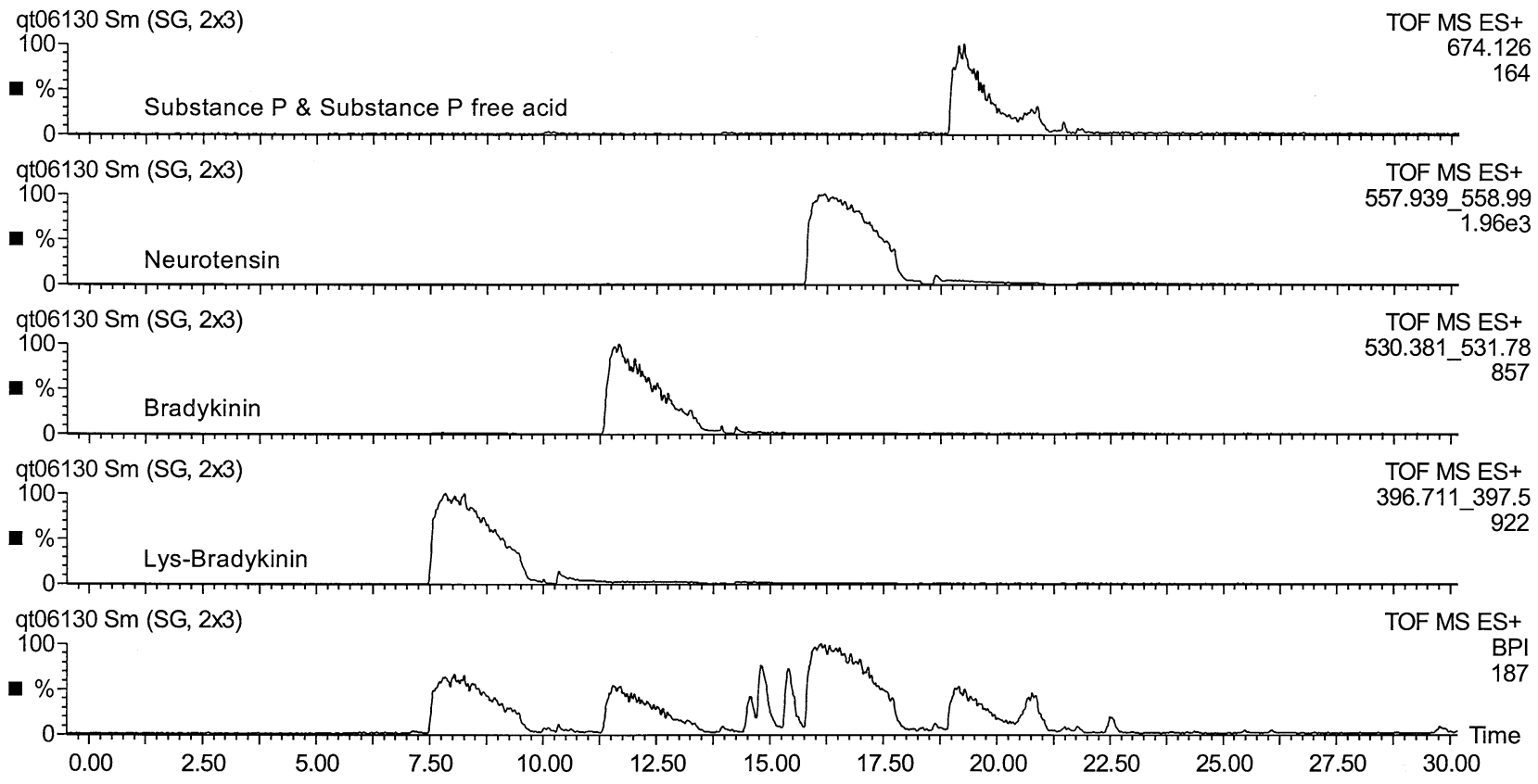

Figure 4. Analysis of the basic peptide mixture shown in Figure 3 under variable flow conditions. The wide chromatographic peaks are due to the decrease flow rate from $200 \mathrm{~nL} /$ minute to $25 \mathrm{~nL} / \mathrm{min}$ as the peak begins to elute into the mass spectrometer. This switching permits an 8 time increase in MS/MS data acquisition time under the chromatographic peak. Note the peptide peaks at 15:00 minutes in the bottom trace (base peak intensity plot), which were analyzed without flow switching. The peak widths of these peptides were not affected by the variable flow switching used to interrogate the two earlier eluting peptides. Their chromatographic resolution was maintained during variable flow mode since the column flow was stopped and the gradient paused during variable flow mode operation.

\section{Data Dependent Scanning}

Two different modes of data dependent scanning were evaluated. In one mode (collision energy profile data dependent scanning), each precursor was interrogated using three different collision energies, with the one to five collision energies used based on the measured $\mathrm{m} / \mathrm{z}$ of the precursor. The user initially defines in the acquisition software sets of precursor $\mathrm{m} / \mathrm{z}$ ranges and assigns three collision energy values to be used to interrogate precursors whose $m / z$ values fall within each range. The collision energies assigned to each $\mathrm{m} / \mathrm{z}$ range are chosen based on the $\mathrm{m} / \mathrm{z}$ values contained within that range and charge states likely to be observed (normally +2 to +4 ), with the goal being that at least one of these values will be close to optimal for that particular precursor. In the second mode (charge state recognition data dependent scanning), a single collision energy was used to interrogate each precursor and was calculated on-thefly based on the observed $\mathrm{m} / \mathrm{z}$ ratio and charge state (also determined on-the-fly). The results given here for the analysis of a tryptic digest of thyroglobulin demonstrate the usefulness of variable flow nanoscale LC/ MS/MS analysis of proteins in proteomics studies. The data dependent scanning parameters of the two different modes and the results (acquired number of MS/MS spectra, number of matched MS/MS spectra, and database score) of the experiment are given in Table 1. As can be seen, the use of charge state recognition-based data dependent scanning gave better results than the use of collision energy profile data dependent scanning and was therefore used for all remaining experiments of this study. The advantages include a 1.8 times increase in the number of acquired spectra, a 1.3 times increase in the number of MS/MS spectra that can be matched back to thyroglobulin, and a 1.7 times increase in "information quality" as measured by increased MASCOT database search scores of individual spectra.

\section{Variable Flow Nanoscale LC/MS/MS Analysis of Mitochondrial Ribosomal Proteins}

Mammalian mitochondrial ribosomes are made up of both a large (39S) and small (28S) subunits [28-30]. The $28 \mathrm{~S}$ subunit was recently reported to be comprised of 29 mitochondrial ribosomal proteins (MRP's) which are present in a 1:1 stoichiometry [30]. To illustrate the applicability of the "peak trapping" variable flow nanoscale LC/MS/MS to the analysis of real-world samples, the method was used to obtain peptide sequence data for mitochondrial ribosomal proteins (MRP's) comprising the $28 \mathrm{~S}$ subunit of the mammalian mitochondrial ribosome [30]. Briefly, 28S subunit was purified from bovine liver and quantitated based on the UV absorbance of bound rRNA. A 10 pmol aliquot of 

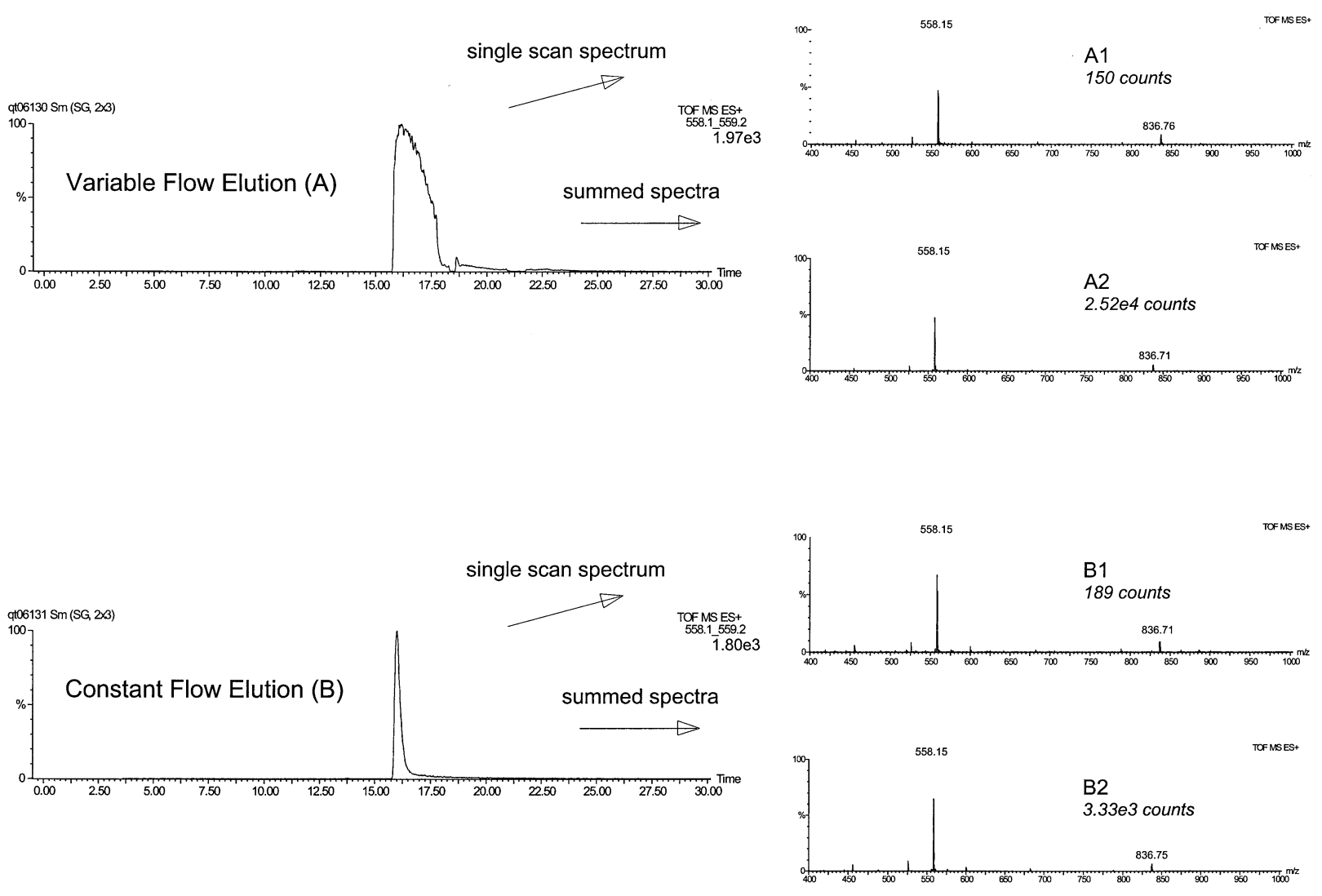

Figure 5. Comparison of variable flow (a) and normal flow (b) LC/MS analysis of neurotensin. The increase in chromatographic peak width (and time available for MS and/or MS/MS acquisition) for the variable flow analysis was 6.4 times. The comparison of a single scan spectrum of the variable flow (A1) and normal flow (B1) experiments show a negligible difference in ion intensity, as expected based on the concentration-sensitive nature of electrospray. The summed spectra across the chromatographic peaks of the variable flow (A2) and normal flow (B2) experiments show a 6.6 times increase in ion abundance in the variable flow mode. This demonstrates that the amount of information that can be assessed from one chromatographic peak can be significantly enhanced using variable flow analysis.

the purified subunit was digested in situ, and subsequently divided into three aliquots containing approximately 3 pmol of digested $28 \mathrm{~S}$ subunit. Aliquots were analyzed by nanoscale LC/MS/MS with and without the use of the variable flow interface. Data-dependent
MS to MS/MS switching was used along with a 3-hour long gradient elution. Data-dependent switching parameters were set up such that up to 8 coeluting precursor ions could be interrogated in a given MS/MS experiment for up to $10 \mathrm{sec}$ per precursor. The identities

Table 1. Evaluation of data dependent scanning acquisition modes for the nanoscale LC/MS/MS analysis of tryptic digested thyroglobuline

\begin{tabular}{|c|c|c|}
\hline & $\begin{array}{l}\text { Collision energy profile data dependent } \\
\text { scanning }\end{array}$ & $\begin{array}{l}\text { Charge state recognition } \\
\text { data dependent } \\
\text { scanning }\end{array}$ \\
\hline Number of collision energies/precursor ion & 3 & 1 \\
\hline Number of precursors/MS scan & 2 & 3 \\
\hline MS acquisition time & $0.5 \mathrm{~s}$ & $0.5 \mathrm{~s}$ \\
\hline MS/MS acquisition time & ( $0.5 \mathrm{~s} \times 3$ different collision energy scans) & $2 s$ \\
\hline Number of MS/MS spectra acquired & 72 & 126 \\
\hline Number of matched MS/MS spectra & 43 & 54 \\
\hline average MASCOT Mowse score & 35 & 69 \\
\hline
\end{tabular}

The sample was analyzed on a $15 \mathrm{~cm} \times 75 \mu \mathrm{m}$ ID nanoscale LC column packed with $3 \mu \mathrm{m}, 100 \AA$ PepMap ${ }^{\mathrm{TM}}$ C18 stationary Phase. Mobile Phase A consisted of $0.1 \%$ formic acid in acetonitrile/water $(2: 98, \mathrm{v} / \mathrm{v})$ and mobile Phase B of $0.1 \%$ formic acid in acetonitrile/water $(80: 20, \mathrm{v} / \mathrm{v})$. The gradient was from $4 \%-32 \% \mathrm{~B}$ in $30 \mathrm{~min}$ and the flow rate equal to $200 \mathrm{nl} / \mathrm{min}$. 


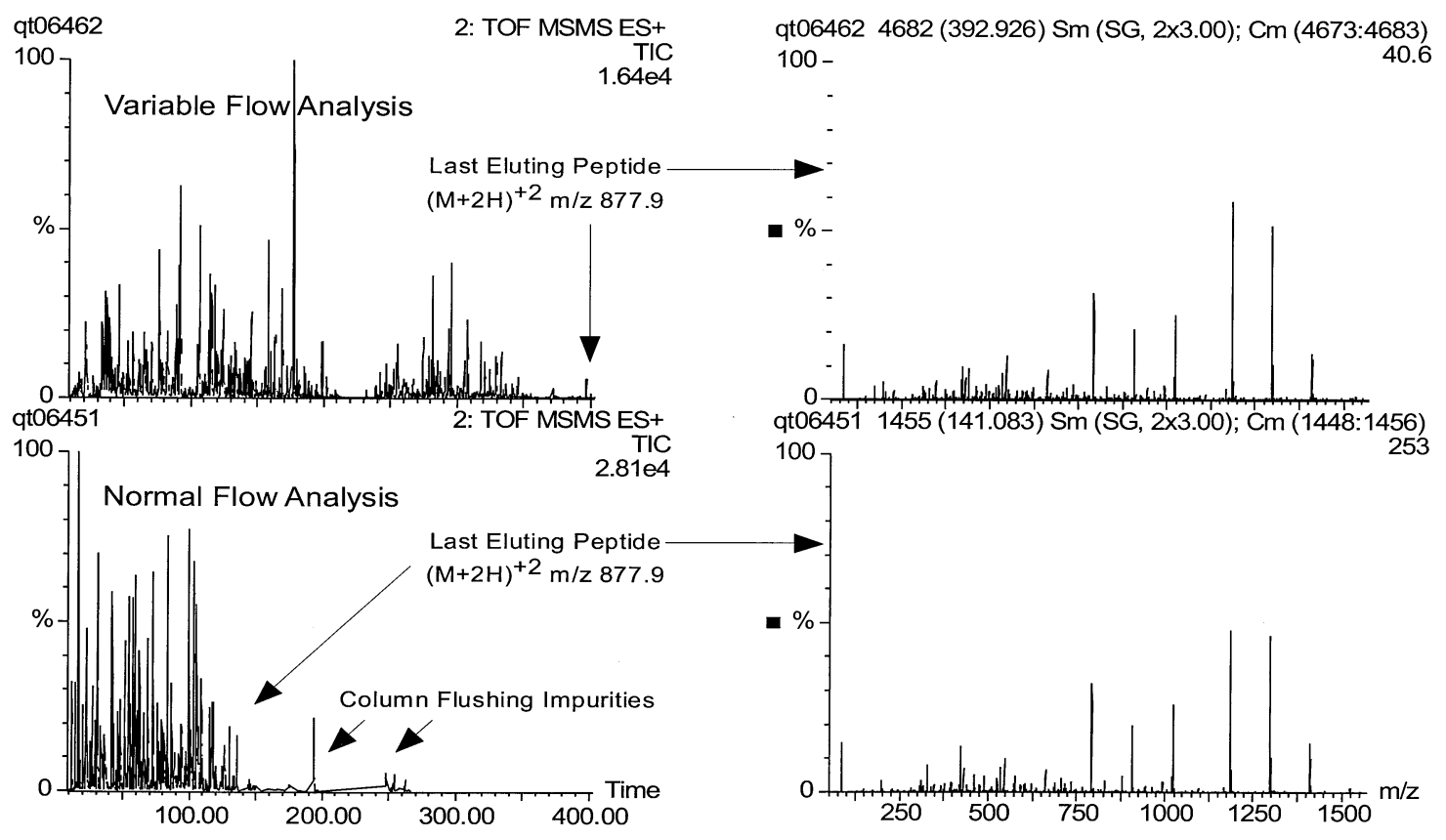

Figure 6. Analysis of total digest of $28 \mathrm{~S}$ mitochondrial ribosome complex under variable flow and normal flow conditions. The use of variable flow increase the total analysis time by approximately 2.8-fold, providing a significantly longer time for acquisition of MS/MS spectra. The last eluting peptide was the same in both analysis. The increased analysis time (6.5 hours) for this peptide under variable flow conditions led to chromatographic band broadening, and a corresponding loss in ion intensity for this very late eluting peptide.

of MRP's obtained from these experiments have been reported elsewhere [28,30], with the purpose of this discussion being the evaluation of variable flow. Data shown in Figure 6 illustrate that the quality of spectra obtained in constant flow and variable flow are comparable. The chromatograms shown on the left are the variable flow total ion chromatogram (A1) and the constant flow total ion chromatogram (B1) for digests of the $28 \mathrm{~S}$ subunit. The MS/MS spectra shown on the right are from the latest eluting peptide in both the variable flow (A2) and constant flow mode (B2). As can be seen, the spectra are essentially identical. Hence, spectral quality is not compromised as a result of the variable flow interface. For this particular peptide, the number of counts was significantly lower in the variable flow mode. This is due in part to the band broadening/peak dilution effect at the extended retention time in variable flow (6 h 33 min vs. 2 h $21 \mathrm{~min}$ ). However, as will be demonstrated later, the overall sensitivity of variable flow is comparable to constant flow with enhanced sequence coverage. Although the overall analysis time in variable flow is increased by a factor of approximately 3 , the variable flow experiment is automated, and no additional operator time is required. As an example, the total ion current (TIC) chromatogram and three of the eight automated MS/MS switching functions (functions two, five and nine) are shown in Figure 7 for the variable flow analysis of the $28 \mathrm{~S}$ complex again. Note in the MS Survey TIC the raggedness of the TIC. This appearance of the TIC is due to a constant elution of peptides during the gradient with a constant switching between the MS and MS/MS scan functions. At approximately $200 \mathrm{~min}$ during this experiment, a temporary glitch with the LC prevented the gradient from progressing until about $225 \mathrm{~min}$. Thus during this time, it is evident from the relatively flat region of the MS Survey TIC that the separation had halted and no further peptides were eluting from the column until the gradient resumed at approximately $225 \mathrm{~min}$. More interesting is the clearly noticeable difference in the individual MS/MS functions, with each "peak" corresponding to MS/MS interrogation of an individual peptide. The individual TICs of the different MS/MS functions also show that, due to the inherent complexity of the Lys-C digested $28 \mathrm{~S}$ small subunit, multiple coelution of the peptides normally occurs throughout the variable flow nanoscale LC process.

The data presented in Figure 8 show some of the spectra that were obtained from the variable flow nanoscale LC chromatogram presented in Figure 7. The top trace (A) is the MS survey scan taken at $135 \mathrm{~min}$. As a result of multiple co-elution, a total of eight MS/MS functions were conducted simultaneously by the mass spectrometer. The resulting MS/MS spectra of the second, fifth and ninth MS/MS function are given in the three lower traces of Figure 8, respectively trace B, C and D. Despite the low number of counts on precursor ion with $m / z$ 755.7, the quality of the MS/MS spectrum was sufficient for automated database searching. Table 2 summarizes the results of the direct analysis of a Lys 

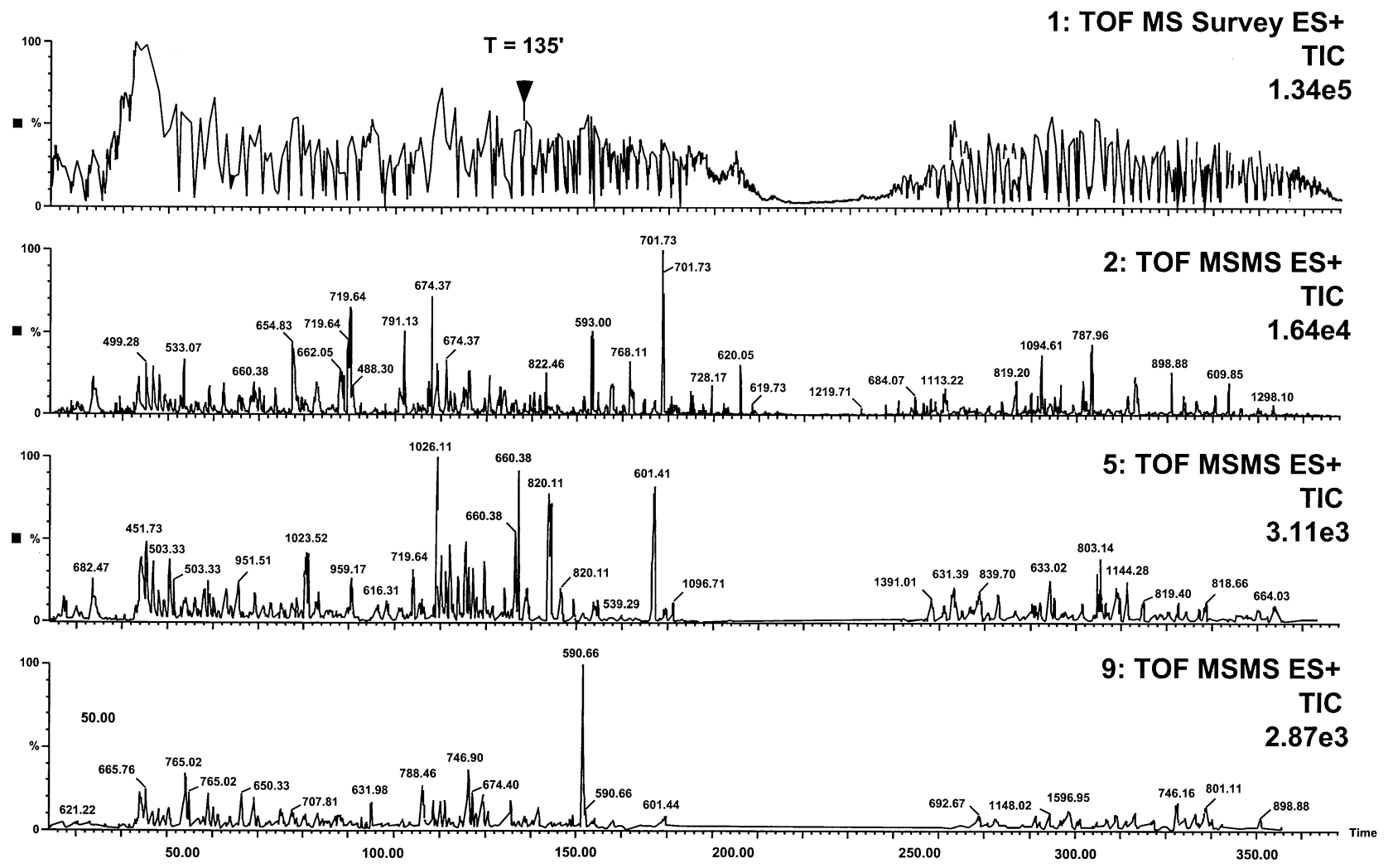

Figure 7. Total ion current (TIC) plots for the variable flow LC/MS/MS analysis of the total digest of the $28 \mathrm{~S}$ complex. Trace 1 is the TIC of the MS survey scans (jagged due to switching between MS and MS/MS). Up to eight precursors were selected form each survey scan for interrogation by MS/MS. Traces 2, 5, and 9 show the TIC of the first, fourth, and eighth MS/MS scan function. Note the high ion current present in the eighth MS/MS scan function, indicating high ion abundance in this scan function for the eighth least abundant ion in the MS survey spectrum. The arrow at $t=135$ minutes indicates the time slice used to generate the spectra shown in Figure 8.

C digest of the small $28 \mathrm{~S}$ protein complex by means of both constant flow and variable flow analysis. The total number of MS/MS spectra that were acquired during the variable flow run was 4,458, of which 1,049 spectra passed the QA filter. Compared to the constant flow analysis, variable flow provided a gain in the total number of acquired MS/MS spectra of 5.7, and a gain in the number of MS/MS spectra passing the QA filter of 1.9. The increase in number of spectra indicates the improvement in proteome coverage possible using variable flow analysis. The number of peptide MS/MS spectra which could be acquired using variable flow significantly exceeds the expected complexity of the $28 \mathrm{~S}$ digest. Thirty-five unique peptides from the 28S MRP complex were observed in the variable flow analysis. (Similar observations were made for the analysis of thyroglobulin.) The number of peptide matches was low relative to number of quality spectra acquired due to several factors: 1) interrogation of multiple charge states from same peptide (providing redundant spectra); 2) most of the $28 \mathrm{~S}$ proteins are novel proteins and not found in the protein database; 3) post-translationally modified peptides; 4) non-specific enzymatic cleavages; 5) use of sample from a species (bovine) under- represented in the protein database (no bovine MRPs in the database). The peptide matches which were observed corresponded to ribosomal proteins in the protein database from human and other (non-bovine) species (which have a low degree of sequence identity due to insertions/deletions, etc.) or corresponded to ESTs. Sufficient MS/MS data was acquired for the identification of the full complement of the expected bovine $28 \mathrm{~S}$ MRPs [23, 27-29], based on either sequence homology to known MRPs from other species, or the in-silico assembly of the full length protein sequence from overlapping ESTs.

\section{Conclusions}

A novel "peak trapping" interface for variable flow nanoscale LC/MS/MS analysis based on postcolumn peak isolation has been demonstrated and utilized for peptide sequencing and protein identification. With the described "peak trapping" interface, the advantages of nanoscale LC were preserved including improved analyte concentration compared to both larger bore LC columns and static nanoelectrospray. In addition, the chromatographic separation on the column was pre- 


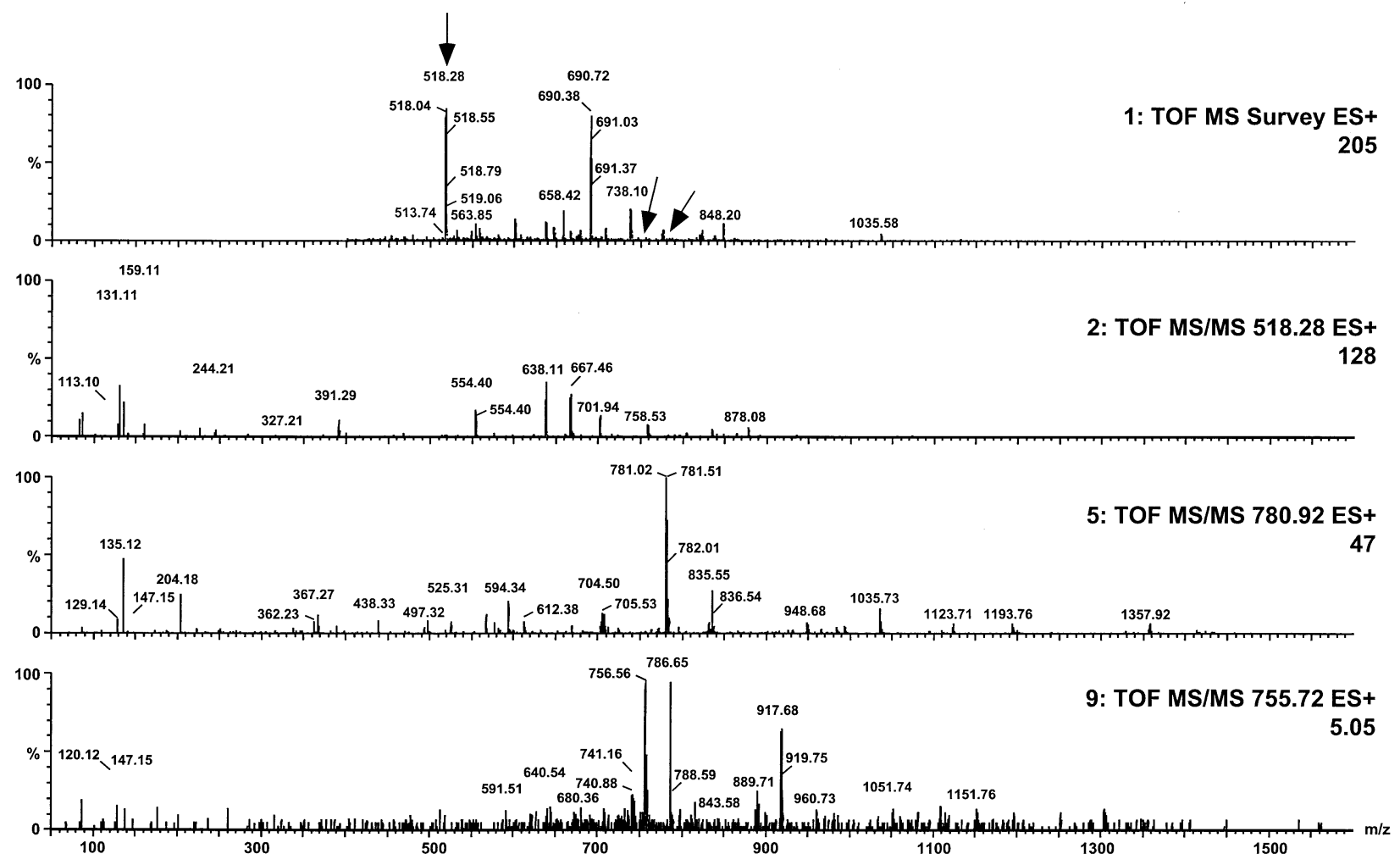

Figure 8. MS (Trace 1) and MS/MS spectra (Traces 2, 5, and 9) of $28 \mathrm{~S}$ complex digest peptides from the time slice $t=135$ minutes from the variable flow LC/MS/MS chromatograms in Figure 7 . The MS data in Trace 1 shows the complexity of the peptide mixture eluting from the nanoscale LC system, and Traces 2, 5 and 9 contain the MS/MS spectra from the most abundant ion $(\mathrm{m} / z$ 518.2), fourth most abundant ion $(\mathrm{m} / \mathrm{z}$ 780.9), and eighth most abundant ion $(\mathrm{m} / \mathrm{z}$ 755.7). The ion current from the eighth most abundant ion was relatively low, but sufficient for database searching.

served and the column not subjected to any pressure shocks due to design of the experimental set-up. The current set-up allows for an increase in MS/MS analysis time by a factor of 8 . However, with very minor modifications longer MS/MS analysis times should be easily achievable. With variable flow nanoscale LC, a six-fold increase in the total number of product ion spectra was obtained compared to constant flow nanoscale LC analysis of a total digest of a mammalian protein complex. This translated into a two-fold increase in the number of "quality" product ion spectra. The difference between total spectra versus quality spectra is most likely due to the fact that the "peak capacity" of the variable flow system was significantly greater than the complexity of the digest, allowing time for the mass spectrometer to switch on spurious signals. By providing a several-fold increase in the number of spectra that can be acquired, this variable flow interface should yield several higher proteome coverage at the peptide level and presumably protein level compared with fixed flow LC/MS/MS analyses.

The results in this paper present some initial experiments that were conducted with the variable flow nanoscale LC interface. Despite a large number of

Table 2. Summary of the results obtained by variable flow nanoscale LC for the analysis of the mammalian $28 \mathrm{~S}$ mitochondrial ribosome complex

\begin{tabular}{lcr}
\hline & $\begin{array}{c}\text { Direct analysis with variable } \\
\text { flow nanoscale LC/MS/MS }\end{array}$ & $\begin{array}{c}\text { Direct analysis with constant } \\
\text { flow nanoscale LC/MS/MS }\end{array}$ \\
\hline \hline Number of MS/MS spectra acquired & 4,458 & 771 \\
Number of spectra after QA filter & 1,049 & 550 \\
Number of total MASCOT matches & 70 & 62 \\
\hline
\end{tabular}

Number of matches observed in both analyses

Number of matches observed in variable flow only

${ }^{a}$ The QA filter is a parameter to discard poor quality spectra during post-acquisition data processing. It takes into account the presence of immonium ions, the intensity of the product ion peaks above precursor mass for multiply charged species, the base peak intensity, and amino acid mass difference between peaks. 
unique software and hardware features, some improvements could potentially be made to enhance the performance of the complete nanoscale LC/MS/MS system. These include: (i) real-time deconvolution of MS precursor ion spectra to preclude the MS/MS acquisition of the same precursor ion from different charge states, (ii) increase in maximum dynamic exclude time in order to prevent the software from repeatedly acquiring MS/MS data on previously interrogated precursors, and (iii) automated (re-) positioning of the spray tip during the "high-flow" and the "low-flow" mode of the variable flow process in trying to achieve the highest possible electrospray signal strength.

\section{Acknowledgments}

The authors would like to thank Robert Bordoli of Micromass Ltd. for his advice and support on the data dependent scanning methods, Mike Bailey of Upchurch Scientific for his kind donation of the 6-port MIV valve, and Rod Davis, GlaxoSmithKline for his help with the development and construction of the LC/MS spray tip mount. Mary Moyer and William Burkhart, GlaxoSmithKline are acknowledged for performing the gel electrophoretic separations and enzymatic protein digestions. Emine Koc and Linda Spremulli, University of North Carolina, prepared and supplied the sample of the $28 \mathrm{~S}$ MRPs.

\section{References}

1. Yates, J. R., 3rd; McCormack, A. L.; Schieltz, D.; Carmack, E.; Link, J. Protein Chem. A 1997, 16, 495-497.

2. Link, A. J.; Eng, J.; Schieltz, D. M.; Carmack, E.; Mize, G. J.; Morris, D. R.; Garvik, B. M.; Yates, J. R., III. Nat. Biotechnol. 1999, 17, 676-682.

3. Washburn, M. P.; Wolters, D.; Yates, J. R., 3rd. Nat. Biotechnol. 2001, 19, 242-247.

4. Moseley, M. A.; Deterding, L. J.; Tomer, K. B.; Jorgenson, J. W. Anal. Chem. 1991, 63, 1467-1473.

5. Hunt, D. F.; Alexander, J. E.; McCormack, A. L.; Martino, P. A.; Michel, H.; Shabanowitz, J.; Sherman, N.; Moseley, M. A.; Jorgenson, J. W.; Tomer, K. B. Tech. Protein Chem. 2 1991, 441-454 [Pap. Annu. Symp. Protein Soc.], 4th.

6. Morris, H. R.; Paxton, T.; Dell, A.; Langhorne, J.; Berg, M.; Bordoli, R. S.; Hoyes, J.; Bateman, R. H. Rapid Commun. Mass Spectrom. 1996, 10, 889-896.

7. Yates, J. R., III; Eng, J. K.; Clauser, K. R.; Burlingame, A. L. J. Am. Soc. Mass Spectrom. 1996, 7, 1089-1098.

8. Perkins, D. N.; Pappin, D. J. C.; Creasy, D. M.; Cottrell, J. S. Electrophoresis 1999, 20, 3551-3567.
9. Opiteck, G. J.; Jorgenson, J. W.; Anderegg, R. J. Anal. Chem. 1997, 69, 2283-2291.

10. Opiteck, G. J.; Lewis, K. C.; Jorgenson, J. W.; Anderegg, R. J. Anal. Chem. 1997, 69, 1518-1524.

11. Opiteck, G. J.; Jorgenson, J. W.; Moseley, M. A., III; Anderegg, R. J. J. Microcolumn Sep. 1998, 10, 365-375.

12. Opiteck, G. J.; Ramirez, S. M.; Jorgenson, J. W.; Moseley, M. A., III. Anal. Biochem. 1998, 258, 349-361.

13. Washburn, M. P.; Wolters, D.; Yates, J. R. Nat. Biotechnol. 2001, 19, 242-247.

14. Davis, M. T.; Stahl, D. C.; Lee, T. D. J. Am. Soc. Mass Spectrom. 1995, 6, 571-577.

15. Davis, M. T.; Lee, T. D. J. Am. Soc. Mass Spectrom. 1997, 8, 1059-1069.

16. Lee, T. D.; Davis, M. T.; Stahl, D. C.; Swiderek, K. M.; Esworthy, R. S.; Hefta, S. A. Nippon Iyo Masu Supekutoru Gakkai Koenshu 1996, 21, 17-32.

17. Martin, S. E.; Shabanowitz, J.; Hunt, D. F.; Marto, J. A. Anal. Chem. 2000, 72, 4266-4274.

18. Shabanowitz, J.; Settlage, R. E.; Marto, J. A.; Christian, R. E.; White, F. M.; Russo, P. S.; Martin, S. E.; Hunt, D. F. Mass Spectrom. Biol. Med. 2000, p 163-177.

19. Davis, M. T.; Stahl, D. C.; Hefta, S. A.; Lee, T. D. Anal. Chem. 1995, 67, 4549-4556.

20. Wilm, M. S.; Mann, M. Int. J. Mass Spectrom. Ion Processes 1994, $136,167-180$.

21. Wilm, M.; Mann, M. Anal. Chem. 1996, 68, 1-8.

22. Burkhart, W. In-Situ Proteolytic Digestions Performed on Proteins Bound to the Hewlett-Packard Hydrophobic Sequencing Cartridge. Academic Press: San Diego, CA, 1993, pp 399-406.

23. Koc, E. C.; Blackburn, K.; Burkhart, W.; Spremulli, L. L. Biochem. \& Biophys. Research Commun. 1999, 266, 141-146.

24. Vissers, J. P. C.; Hulst, W. P.; Chervet, J. P.; Snijders, H. M. J.; Cramers, C. A. J. Chrom. B: Biomedical Applications 1996, 686, $119-128$.

25. Geromanos, S.; Freckleton, G.; Tempst, P. Anal. Chem. 2000, 72, 777-790.

26. Moseley, M. A.; Blackburn, R. K.; Burkhart, W.; Vissers, J. P. C.; Bordoli, R. S. Increased Proteome Coverage using a Novel Peak Trapping Interface for Nanoscale Capillary LC/MS/MS Analysis of Complex Peptide Mixtures. Proceedings of the 48th ASMS Conference, Long Beach, CA, 2000.

27. Yamashita, M.; Fenn, J. B. J. Phys. Chem. 1984, 88, 4451.

28. Koc, E. C.; Burkhart, W.; Blackburn, K.; Moseley, A.; Koc, H.; Spremulli, L. L. J. Biol. Chem. 2000, 275, 32585-32591.

29. Koc, E. C.; Burkhart, W.; Blackburn, K.; Moyer, M. B.; Schlatzer, D. M.; Moseley, A.; Spremulli, L. L. J. Biol. Chem. 2001, 276, 43958-43969.

30. Koc, E. C.; Burkhart, W.; Blackburn, K.; Moseley, A.; Spremulli, L. L. J. Biol. Chem. 2001, 276, 19363-19374. 